\title{
SILICA DIOXIDE NANOFIBER BASED DRUG DELIVERY SYSTEM WITH SUSTAINED RELEASE
}

\author{
${ }^{1}$ Hana TOMÁNKOVÁ, ${ }^{1,2}$ Miroslava RYSOVÁ, ${ }^{3}$ Marie SCHNEIDROVÁ, ${ }^{3}$ Hana SKLENÁŘOVÁ \\ 1Institute for Nanomaterials, Novel Technologies and Innovation (CXi), TUL, Liberec, Czech Republic, EU, \\ hana.tomankova@tul.cz \\ 2Institute of New Technologies and Applied Informatics (FM), TUL, Liberec, Czech Republic, EU \\ ${ }^{3}$ Faculty of Pharmacy in Hradec Králové, Charles University, Czech Republic, EU
}

https://doi.org/10.37904/nanocon.2019.8770

\begin{abstract}
We developed a transdermal drug delivery system containing tetracycline as a model antibiotic for wound healing application. Our previous in vitro tests proved no cytotoxic effect nor antibacterial activity of the silica nanofiber based matrix. This study was designed to evaluate biocompatibility of our nanofiber-antibiotic system via reconstructed human skin model, and to confirm sustained release of the incorporated active compound. Further characterization showed that both moisture vapor permeability and enhanced hydrophilicity of the modified nanofibers remained consistent after functionalization. The summarized results proved that this drug delivery system is a perspective solution in potential medicated sustained release patch for wound healing.
\end{abstract}

Keywords: Silica dioxide nanofibers, sustained release, wound healing, antibiotic, topical delivery.

\section{INTRODUCTION}

Skin diseases are global problem worldwide including chronic wounds affecting about $1-2 \%$ of the population in the developed countries [1,2]. Bacterial contamination is one of the main reasons of difficult-to-healing wounds development, resulting in need for therapies based on broad-spectrum antibiotics [3]. Unfortunately, conventional oral or intravenous administrations may lead to systemic toxicity, bacterial resistance or other side effects. To overcome this problem, many researchers focus their attention to develop novel drug delivery systems with sustained release of the active compound directly into the wound [4,5]. Due to their unique physical and chemical properties, especially the high specific surface and excellent vapour permeability combined to the microbe-resistant membrane structure, nanofibers represent a robust material for this biomedical application [6]. Besides the commonly used natural and synthetic polymers, nanofibers can also be fabricated of inorganic compounds. We have focused on silica dioxide for its better biocompatibility in comparison to other metal oxides and low immunogenicity resulting from the fact that the silica is natural for the body [7]. Moreover, their surface is suitable for common chemical modifications necessary for subsequent drug conjugation $[8,9]$.

We have previously demonstrated the antibacterial activity as well as the biocompatibility in vitro of the silica dioxide nanofibers functionalized by silanization and subsequent bonding of tetracycline [10]. In this paper we present results of the biocompatibility tests performed via skin irritation test and aim to show the sustained release of the model antibiotic (tetracycline). Finally, we have focused on verifying hydrophilicity and moisture vapour permeability of the functionalized fibers.

\section{MATERIALS AND METHODS}

\subsection{Chemicals}

Tetraethyl orthosilicate (TEOS, silica fibers precursor), (3-aminopropyl)triethoxysilane (APTES, the coupling agent) were purchased from Sigma-Aldrich (St. Louis, MO, USA). The remaining chemicals, all of analytical reagent grade, were supplied by Penta (Prague, Czech Republic). 


\subsection{Preparation of nanofibers}

The silica nano-fibrous sheets of surface density of $470 \mathrm{~g} / \mathrm{m}^{2}$ were electrospun under stable conditions, at $22{ }^{\circ} \mathrm{C}$ (air-conditioned space) using the NanoSpider device (NS 1WS500U, Elmarco Ltd.). The distance between an electrode and a collector was $175 \mathrm{~mm}$. The spinning solution was prepared by sol-gel method using TEOS as a precursor, voltage of $70 \mathrm{kV}$ was applied. Finally, electro-spun sheets were thermally treated $\left(180{ }^{\circ} \mathrm{C}\right)$ to ensure their prolonged stability. Surface functionalization was realized by silanisation [11] as nanofibrous mats were cut into $5 \times 5 \mathrm{~cm}$ sheets and immersed into $3 \%(\mathrm{v} / \mathrm{v})$ APTES solution containing $4 \%$ of water in ethanol $(\mathrm{v} / \mathrm{v})$. The $\mathrm{pH}$ was adjusted to 5.3 using acetic acid. After 2-hour silanization of nanofibers at a laboratory temperature under continual shaking, the samples were washed by the solution containing $4 \%$ water in ethanol $(\mathrm{v} / \mathrm{v})$. Finally, the nanofibers were dried at $110{ }^{\circ} \mathrm{C} / 30 \mathrm{~min}$. Control samples were prepared evenly but immersed in the mixture water/ethanol (4/96) with pH 5.3 only, without APTES.

\subsection{Reconstructed human skin model}

Biocompatibility was evaluated via skin irritation test on reconstructed ex vivo skin models of epidermis Epiderm $^{\mathrm{TM}}$ (MatTek Corporation, Ashland, USA). The test was carried out in accordance with the instructions given in the MK-24-007-0023 IN VITRO EpiDerm ${ }^{\text {TM }}$ SKIN IRRITATION TEST (EPI-200-SIT) and OECD Guideline No. 1 test. 439 - In vitro skin irritation: Reconstructed human epidermis test method, OECD Guideline for Chemicals testing 431 - In vitro skin corrosion: Human skin model test and Method B.46 - In vitro skin irritation: Reconstructed human epidermis model test 761/2009. In the test, living tissue of the epidermis was exposed to test materials with potential for skin applications and drug delivery. In accordance with the protocol MK-24-007-0023, exposure to the tested nanofibrous layers was performed in a size equivalent to the surface area of the skin model. Exposure lasted 60 minutes and was followed by 44 hours incubation under standard conditions without test sample. The assay was then evaluated by evaluation of active metabolic conversion of 3- (4,5-dimethylthiazol-2-yl) -2,5-diphenyltetrazolium (MTT).

\subsection{Release kinetic testing}

In vitro release studies were done using automated Franz diffusion cell apparatus coupled to low pressure chromatographic system [12]: Franz cell (PermeGear, Hellertown, USA) consisted of donor and acceptor compartments between which the mixed cellulose esters membrane (Merck KGaA, Darmstadt, Germany) was placed as a barrier. The experiments were conducted in three independent vertical Franz cells with a nominal volume of acceptor compartment of $15 \mathrm{ml}$ and the exposed membrane surface area of $3,14 \mathrm{~cm}^{2}$. The nanofiber sample was placed on the cellulose membrane from the compartment side of the cell. The acceptor compartment was filled with ultrapure water $(\mathrm{pH} 4,5)$ maintained at $37^{\circ} \mathrm{C}$ by a water bath in order to ensure the temperature of $32{ }^{\circ} \mathrm{C}$ on the surface of the membrane. These conditions were established to mimic the physiological conditions in the wound. The acceptor medium was stirred continuously. For each analysis, $100 \mu \mathrm{L}$ of sample were removed every 15 minutes, injected into HPLC column and spectrophotometrically quantified at $365 \mathrm{~nm}$. The receptor compartment was topped off with fresh medium after each sampling. The release profile for each sample was followed for 8 hours.

\subsection{Moisture vapour permeability}

The change in the moisture vapour permeability of the test samples in the presence of test solution was determined according to the European Standard EN13726-2: test methods for primary wound dressings [13]. Sample of each fibrous sheet was applied to a Paddington cup containing $25 \mathrm{ml}$ of test solution. The cup was then weighed and placed in an incubator at $37^{\circ} \mathrm{C}$. A tray containing $1 \mathrm{~kg}$ of freshly dried silica gel was placed in the bottom of the incubator to maintain a low relative humidity within the chamber. At determined time period, the entire system of the cup was weighed and the weight of moisture vapour loss through the nanofibers was calculated. 


\subsection{Water contact angle measurement}

To study the hydrophilicity/hydrophobicity rate, the contact angle was measured at different positions on the samples at room temperature using a Kruss Drop Shape Analyzer DS4. A total volume of $2 \mu \mathrm{L}$ of distilled water was dropped on the surface of a dry membrane surface, and the average values of the contact angle were calculated.

\section{RESULTS}

\subsection{Biocompatibility via reconstructed human skin model}

EpiDerm ${ }^{\mathrm{TM}}$ is a multilayer tissue model consisting of primary human fibroblasts and keratinocytes allowing biocompatibility evaluation in the liquid /air interface and was validated in 2018 as a human relevant alternative to the in vivo skin irritation test. The results summarized in the Figure 1 show that none of the tested materials can be considered as irritating. In this test group, the viability of the models was evaluated to be significantly greater than $50 \%$ (Cut-off level). The viability significantly exceeded $90 \%$ in all samples and reached level of viability of the control (untreated epidermis model. In the case of nanofibers with immobilized antibiotic the average value was $101.3 \pm 2.4 \%$ of NC viability.

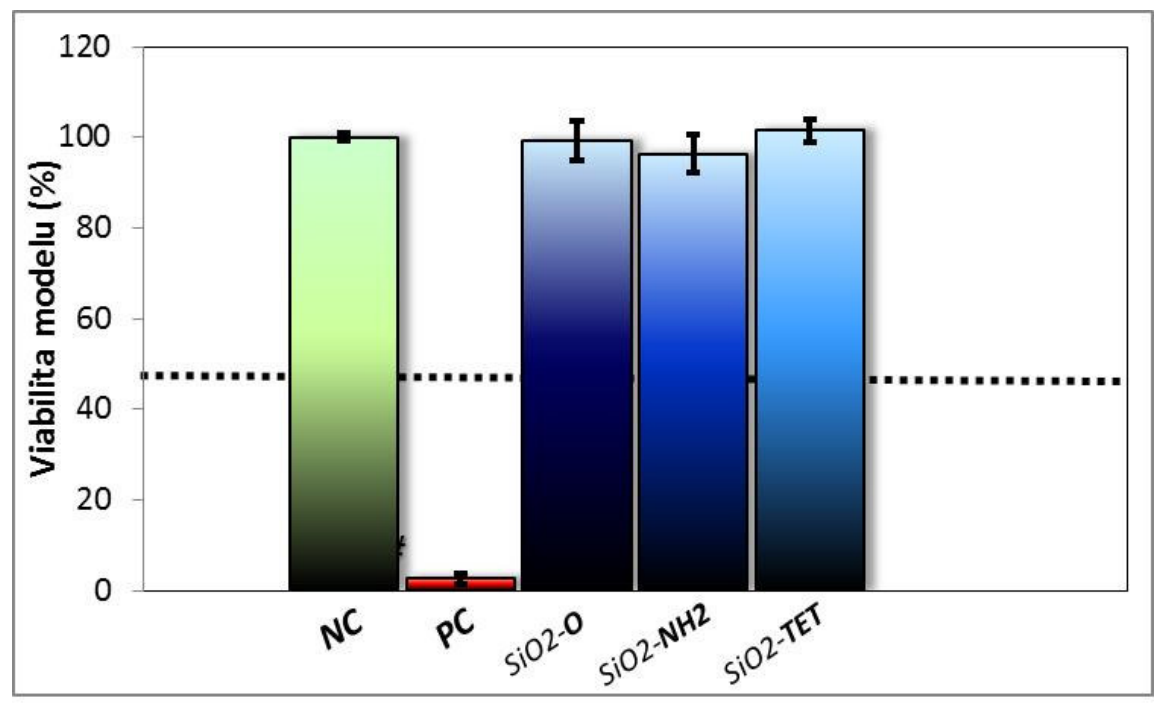

Figure 1 Results of in vitro skin irritation test on reconstructed human epidermis EpiDerm ${ }^{\mathrm{TM}}$ model (SiO2-O are the untreated nanofibers, SiO2-NH2 the nanofibers after surface functionalization and SiO2-TET the silanized nanofibers with further functionalization with antibiotic tetracycline.

\subsection{Release kinetics study}

Franz diffusion cell based experiments are emerging as a generally accepted in vitro release testing method for semisolid formulations [14]. These open chambre based studies can simulate a topical application of the tested formulation by the mean of synthetic membrane, a tissue construct or a biological construct placed between the sample and the acceptor medium. The system is then used to evaluate the penetration of a drug through the barrier immiting the skin. The Figure 2 summerizes both release profiles of the nanofibers conjugated to tetracycline with or without the amino groups incorporated on the surface. As the data show, during the monitored period, the amount of the antibiotic in the medium increases much more slowly for the sample of nanofibers that carry the incorporated amino groups on their surface. The tested hypothesis about the benefit of the silanization step before antibiotic conjugation on the sustained release of the drug is therefore supported by the obtained data. We have also tested different temperature conditions and have observed that 
the increase of the temperature led to accelerated drug release (unpublished data). This situation simulates environment of a contaminated wound undergoing inflammation leading to temperature rise. Over the next months, additional measurements will be taken to monitor the release of the substance in days, not just hours. However, we are aware of the necessity of parallel testing of degradation products of tetracycline in the medium due to the long-term study and the testing conditions that are degrading for the antibiotic under test.

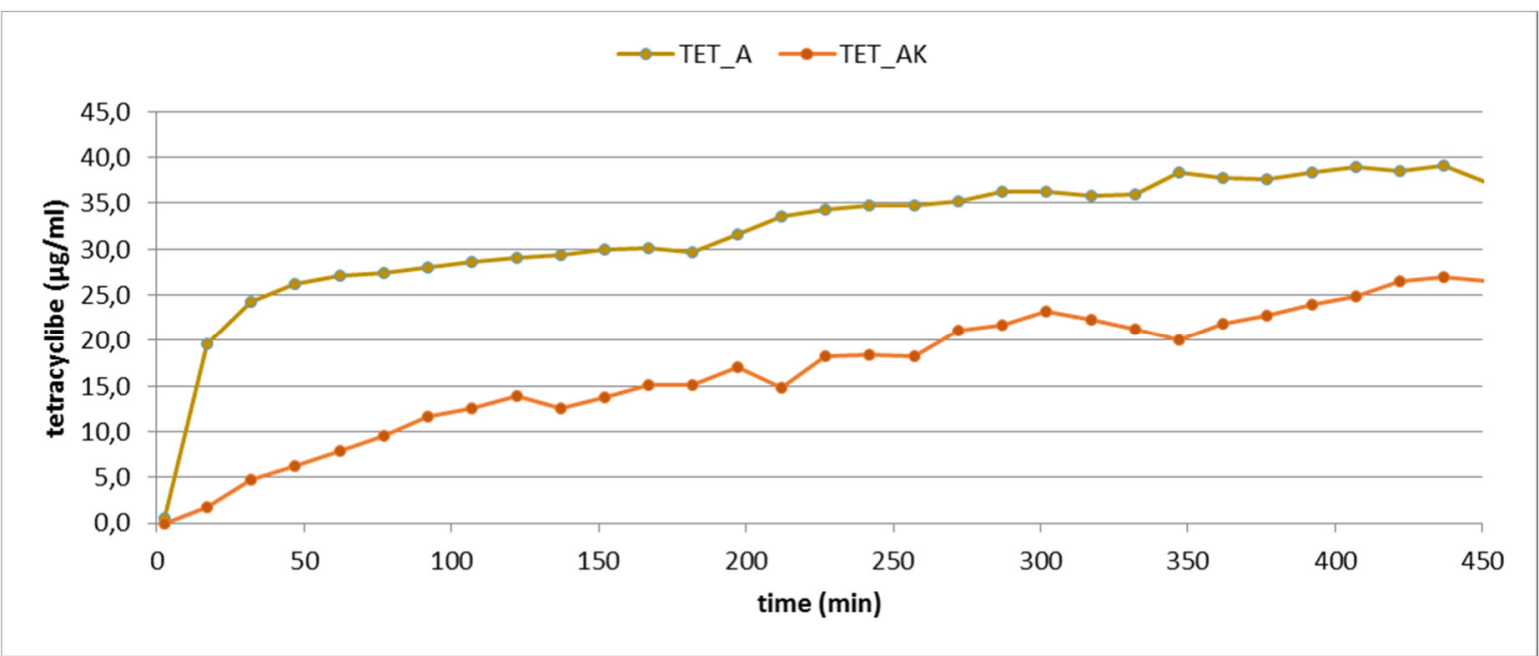

Figure 2 Release kinetic profiles for pristine $\mathrm{SiO} 2$ nanofibers with adsorbed tetracycline (TET_A) and for silanized $\mathrm{SiO} 2$ nanofibers conjugated to tetracycline (TET_AK), under testing conditions of $32{ }^{\circ} \mathrm{C}, \mathrm{pH} 4.5$, HAWP membrane

\subsection{Moisture vapor permeability testing}

Another important parameter observed usually in skin covers is the ability to remove water vapor, i.e. moisture vapor permeability. The proposed nanofibrous systems have also been tested for this property using the standardized Paddington Cup method (The European Standard EN13726-2, Test methods for primary wound dressings - Part 2: Moisture vapour transmission rate of permeable film dressings). As can be seen from the Figure 3, the water vapor permeability of nanofiber samples has been maintained after functional surface treatments and is similar to the commonly used, certified polypropylen/viscose based patch cushion.

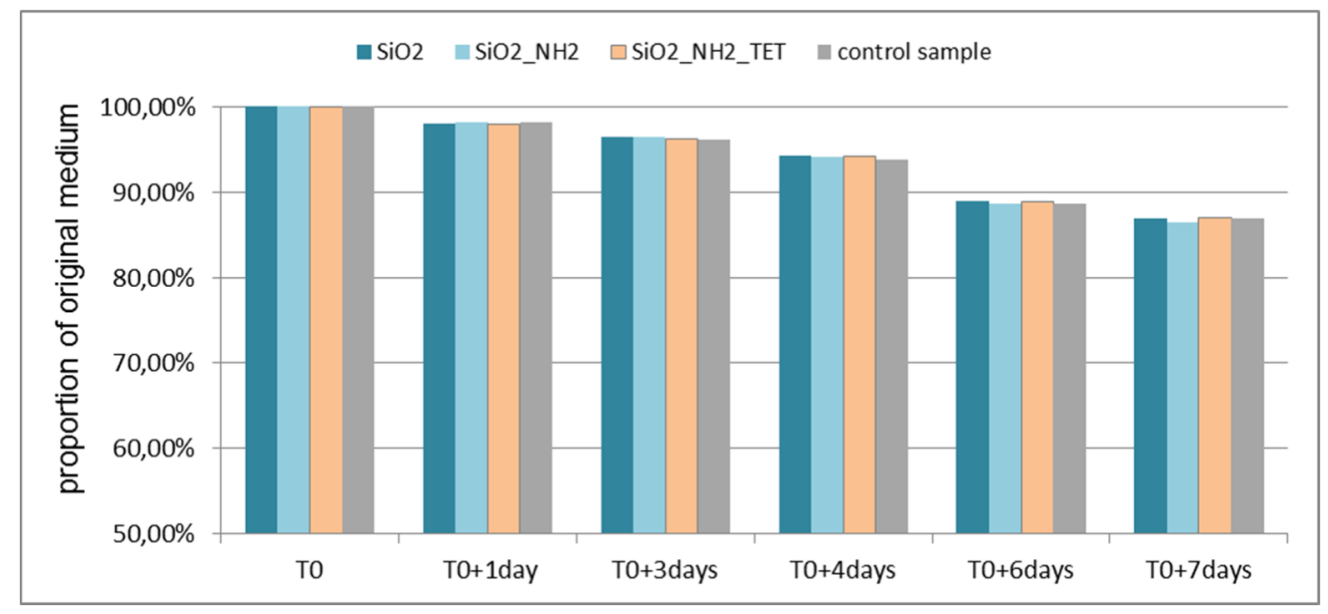

Figure 3 Monitoring of water vapor leakage from Paddington Cup system through various type of materials: silica dioxide nanofibers non-treated ( $\mathrm{SiO} 2)$, silanized ( $\mathrm{SiO} 2 \mathrm{NH} 2)$, silanized/tetracycline conjugated (SiO2_NH2_TET) and commonly used viscose/polypropylene based patch cushion as a control sample 


\subsection{Water contact angle measurement}

An important property of materials considered for transdermal applications is their hydrophilicity. The basic prerequisite for proper wound healing is the possibility of draining excess exudate from the wound while maintaining the so-called moist healing if possible. Therefore, all considered materials were also evaluated from this point of view by measuring the contact angle of wetting on a drop sessile to the surface of the tested nanofibrous material. Figure 4 shows that hydrophilicity of the nanofibers has improved with the surface treatments: the surface of the material changed from very hydrophobic (water contact angle around $120^{\circ}$ ) to completely hydrophilic $\left(0^{\circ}\right)$.

\begin{tabular}{|c|c|c|c|}
\hline Type of Nanofibers & $\begin{array}{l}\text { droplet contact } \\
\text { to }\end{array}$ & $\begin{array}{l}\text { droplet contact time: } \\
\qquad \text { to }+1 \text { min }\end{array}$ & $\begin{array}{l}\text { droplet contact time: } \\
\qquad 0+5 \mathrm{~min}\end{array}$ \\
\hline $\mathrm{SiO}_{2}$ & $=$ & $=\quad-{ }^{117}$ & $={ }^{118,3^{*}}$ \\
\hline $\mathrm{SiO}_{2} \mathrm{NH}_{2}$ & 。 & & \\
\hline $\mathrm{SiO}_{2-} \mathrm{NH}_{2} \mathrm{TET}$ & & & \\
\hline
\end{tabular}

Figure 4 The size of the contact wetting angles on a drop adjacent to the surface of different types of nanofibers, measured in different time ranges between the drop and sensing ( $0 \mathrm{~min}, 1 \mathrm{~min}, 5 \mathrm{~min})$

\section{CONCLUSION}

Our results represented in this study have proven silica nanofibers to be an interesting drug delivery solid formulation for treatment of contaminated topical wounds. The biocompatibility test performed on reconstructed human epidermis model EpiDerm ${ }^{\mathrm{TM}}$ in vitro has shown neither cytotoxicity nor irritant effect of silica nanofibers with or without immobilized tetracycline. The release kinetics analysis has shown impact of state of the wound on the released antibiotic quantum as the TET release was decreased relevantly through the HAWP membrane simulating skin (epidermis) barrier under $32{ }^{\circ} \mathrm{C}$ in T-AK samples proving the positive effect of the silanization procedure on the drug immobilization and its subsequent release. We have also demonstrated that changed surface energy or wettability had no significant effect on the vapour permeability within the seven days period, which is an important parameter of wound dressing applicability.

\section{ACKNOWLEDGEMENTS}

Realization and presentation of the research reported in this paper was supported by the TAČR project TH02020786, the MPO Trio project FV10605 realized at the Institute for Nanomaterials, Novel Technologies and Innovation of the Technical University of Liberec and by the Ministry of Education,

Youth and Sports of the Czech Republic and the European Union - European Structural and Investment Funds in the frames of Operational Programme Research, Development and Education project Hybrid Materials for Hierarchical Structures (HyHi, Reg. No.

CZ.02.1.01/0.0/0.0/16_019/0000843) 


\section{REFERENCES}

[1] CHEREDDY, K. K., LOPES, A., KOUSSOROPLIS, S. Combined effects of PLGA and vascular endothelial growth factor promote the healing of non-diabetic and diabetic wounds. Nanomedicine Nanotechnology Biology and Medecine. 2015. vol.11(8), pp. 1975-1984.

[2] GAINZA, G., VILLULAS, S., PEDRAZ, J. L., HERNANDEZ, R. M., IGARTUA, M. Advances in drug delivery systems (DDSs) to release growth factors for wound healing and skin regeneration. Nanomedicine Nanotechnology Biology and Medecine. 2015. vol.11(6), pp. 1551-1573.

[3] RAYMENT, E. A., DARGAVILLE, T. R., SHOOTER, G. K., GEORGE, G. A., UPTON, Z. Attenuation of protease activity in chronic wound fluid with bisphosphonate-functionalised hydrogels. Biomaterials. 2008. vol. 29(12), pp. 1785-1795.

[4] HUA, S. Lipid-based nano-delivery systems for skin delivery of drugs and bioactives. Frontiers in Pharmacology. 2015. vol. 6, pp. 219

[5] PACHUAU, L. Recent developments in novel drug delivery systems for wound healing. Expert Opinion on Drug Delivery. 2015. vol.12(12), pp. 1895-1909.

[6] CHEN, S., LIU, B., CARLSON, M.A., GOMBART, A.F., REILLY, D.A., XIE, J. Recent advances in electrospun nanofibers for wound healing. Nanomedicine. 2017. vol.12(11), pp. 1335-1352.

[7] MARTIN, K. R. The chemistry of silica and its potential health benefits. The Journal of Nutriotion Health and Aging. 2007. vol.11(2), pp. 94-97.

[8] TOMÁNKOVÁ, H., ROTKOVÁ, J. and RYSOVÁ, M. Inorganic Silica Nanofibers in a Role of Bioactive Compound Carrier. TANGER Ltd. Nanocon. 2015

[9] DANILOVÁ, I. et al. 2014. Immobilization of esterase enzyme onto silica nanofibers for biomedical applications. Vlákna a textil. 2014. vol.21(2), pp. 3-11.

[10] TOMÁNKOVÁ, H., RYSOVÁ, M., PAZOUREK, A. Plasma Treatment in Suface Modification of Silica Dioxide Nanofibers. TANGER Ltd. Nanocon 2018.

[11] HERMANSON, G. T. Silane Coupling Agents. In: Bioconjugate Techniques (Second Edition). Academic Press, New York, USA, 2008. Chapter 13, pp. 562-581.

[12] ALVES, C. A., RAMOS, I. I. On-line automated evaluation of lipid nanoparticles transdermal permeation using Franz diffusion cell and low-pressure chromatography. Talanta. 2016. vol.146, pp. 369-374.

[13] British Standards Institute - BSI. (2002). BS EN 13726-1:2002: test methods for primary wound dressings. Part 2: Moisture vapour transmission rate of permeable film dressings. London: BSI.

[14] LIU, D., ZHANG, C., ZHANG, X., WANG, P., LI, J., YI, D., JIN, Y., YANG, D. Permeation measurement of gestodene for some biodegradable materials using Franz diffusion cells. Saudi Pharmaceutical Journal. 2015. vol.23(4), pp. 413-420 